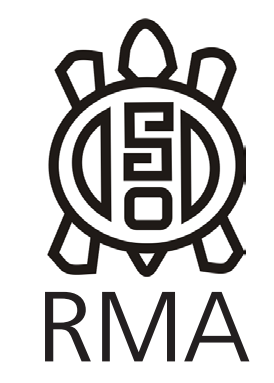

VI Jornadas

Arqueológicas Cuyanas

\section{Fuente de obsidiana El Peceño: primeros resultados de su abordaje tecnológico, geoquímico y espacial}

\author{
Obsidian source El Peceño: first results of \\ technological, geochemical and spatial approach
}

"MHNSR, CONICET-IANIGLA Grupo Vinculado San Rafael. E-mail: mpompei@mendoza-conicet.gob.ar *International Center Earth Science-Malargüe. E-mail: Isalgan@mendoza-conicet.gob.ar

\begin{abstract}
Resumen
La fuente primaria de obsidiana El Peceño se encuentra ubicada en la planicie oriental del sur de Mendoza y su uso ha sido vinculado a contextos arqueológicos del Holoceno Tardío. Su ubicación geográfica, accesibilidad y calidad para la talla le otorgan características ventajosas en relación con otras fuentes de obsidiana conocidas. Sin embargo, su dispersión geográfica es menor que la registrada en las fuentes de obsidiana de cordillera. Estudios geoquímicos previos hicieron posible discriminar dos sub-fuentes denominadas El Peceño-1 y El Peceño-2. Recientes muestreos de campo permitieron ubicar espacialmente los afloramientos de ambas sub-fuentes y los talleres de procesamiento y extracción. En este trabajo se presenta la caracterización espacial y geoquímica de las sub-fuentes taller, sus particularidades macroscópicas y las primeras tendencias de su abordaje tecnológico. Los resultados muestran diferencias en la distribución espacial de las sub-fuentes y en las actividades de producción representadas en las variedades de El Peceño. La obtención de lascas y la confección de preformas serían actividades representadas en EI Peceño-1, mientras que el descortezamiento y formatización de núcleos son frecuentes en El Peceño-2. Por último, se proponen expectativas del modo de transporte de estas variedades de obsidiana y las perspectivas de trabajo futuro.
\end{abstract}

Palabras clave: obsidiana; tecnología lítica; estudios geoquímicos; fuentes de aprovisionamiento; sur de Mendoza.

\begin{abstract}
The primary source of obsidian El Peceño is located in the eastern plains of southern Mendoza and its use has been linked to archaeological contexts of late Holocene. Its geographical location, accessibility, and quality, provide advantageous characteristics of procurement when compared to other known sources of obsidian. However, the geographic dispersion of this source is narrower compared with other Andean obsidian sources. Previous geochemical studies permitted discriminating between two sub-sources called El Peceño-1 and El Peceño-2. Recent field sampling allowed locating outcrops of both sub-sources and of processing and extraction locations. In this paper, spatial and geochemical characterization of the sub-sources, their macroscopic particularities, and first trends of their technological analysis are presented. The results show differences in distribution spatial of the sub-sources and production activities corresponding to different varieties of El Peceño obsidian. Flake and preform production were probably the activities conducted at El Peceño-1, while cortex removal and core formalizing, were the main activities conducted at El Peceño-2. Finally, expectations for the most frequent mode of transportation of these varieties of obsidian and the perspectives for future work are presented.
\end{abstract}

Keywords: obsidian; lithic technology; geochemical studies; sources of procurement; southern Mendoza.

La obsidiana es una de las materias primas seleccionadas por las poblaciones cazadoras recolectoras para la confección de herramientas líticas por sus aptitudes para la talla (Eerkens et al. 2009; Ericson 1977; Summerhayes et al. 2013). Su registro en el sur de Mendoza presenta un incremento hacia el Holoceno tardío que fue interpretado como parte de un aumento en las relaciones de interacción (Lagiglia 2002; Neme y Gil 2008, 2012). Las áreas andina y extra andina del Centro Occidental Argentino y Nordpatagonia presentan un importante número de fuentes de obsidiana primarias y secundarias (Barberena et al. 2011; Cortegoso et al. 2012; Durán et al. 2004; Giesso et al. 2011; Seelenfreund et al. 1996; Salgán et al. 2015, entre otros). En los últimos 20 años,

Recibido 06-03-2016. Recibido con correcciones 22-06-2016. Aceptado 23-07-2016

Revista del Museo de Antropología, Suplemento Especial 1: 51-58, 2017 / ISSN 1852-060X (impreso) / ISSN 1852-4826 (electrónico) 
trabajos de investigación macro regionales han permitido localizar más de seis fuentes naturales de obsidiana y aún existen señales geoquímicas de ubicación desconocida, así como áreas no exploradas (Figura 1A). El avance actual de las investigaciones muestran un uso diferencial de los afloramientos de cordillera (Las Cargas, Laguna del Maule y Laguna El Diamante-Paramillos) y planicie (El Peceño y Cerro Huenul), destacándose que las fuentes cordilleranas exhiben una dispersión temporal y espacial mayor que las de planicie (Barberena et al. 2011; Cortegoso et al. 2012; Giesso et al. 2011; Salgán et al. 2015). Estos abordajes estuvieron centrados en estudios geoquímicos y se propusieron conocer la diversidad de fuentes de obsidiana utilizadas en la macro región, ubicar su procedencia y estimar su distribución espacial y temporal (Cortegoso et al. 2012; Durán et al. 2004; Giesso et al. 2011; Neme y Gil 2008, 2012). Sin embargo, como plantean Cortegoso et al. (2012), es necesario resolver los problemas metodológicos de los muestreos de artefactos arqueológicos, considerar los aspectos tecnológicos e incorporar en estos análisis las áreas y bloques temporales de los que se carece de información. Estos aspectos han comenzado a ser abordados en las distintas áreas (Cortegoso et al. 2016; Salgán et al. 2012a, 2014, 2015).

La fuente de obsidiana El Peceño (Figura 1B y C), es mencionada por primera vez hace más de diez años por Durán et al. (2004). Sin embargo, su localización primaria y caracterización de los subgrupos geoquímicos El Peceño-1 y El Peceño-2 se dieron a conocer más recientemente por Giesso et al. (2011). En dicho trabajo se presenta una cuantiosa base de datos geoquímicos de muestras arqueológicas y de fuentes naturales, lo que permitió discriminar ambos subtipos de obsidiana El Peceño y estimar su dispersión espacial y temporal. Los autores proponen que El Peceño comprende un área de dispersión máxima de entre 100 y 150 km y una explotación efectiva en el último milenio, con un registro de uso inicial hacia la segunda mitad del Holoceno tardío (Giesso et al. 2011). La explotación efectiva de la fuente El Peceño se daría al tiempo en que, en la planicie oriental, se registra la máxima variabilidad de fuentes de obsidiana representadas, incluyendo las fuentes minoritarias extraregionales. Este fenómeno pudo haber estado relacionado con la ampliación de los rangos de movilidad o con el establecimiento de redes de intercambio e interacción a gran escala. Tendencias que abren interrogantes en cuanto a la baja intensidad de uso local y regional de los subtipos de obsidiana El Peceño, pese a sus ventajas de accesibilidad en relación con las fuentes cordilleranas (Cortegoso et al. 2012; Giesso et al. 2011; Neme y Gil 2008, 2012; Salgán et al. 2012a).

En este marco, y como parte de un proyecto mayor que propone el estudio sistemático de las fuentes de obsidiana del sur de Mendoza, se presentan los primeros resultados de los trabajos Ilevados a cabo en El Peceño. Se parte de considerar a las fuentes como un espacio más de actividad humana que implica múltiples episodios de extracción y producción de artefactos tallados, constituyendo un paisaje lítico (Gould y Saggers 1985). El objetivo de este trabajo es detallar las herramientas metodológicas utilizadas para el abordaje espacial y tecnológico de la fuente de obsidiana, y delinear los resultados preliminares. Estos permiten esbozar tendencias tecnológicas para el uso de las dos variedades de obsidiana bajo estudio.

\section{Fuente de obsidiana El Peceño}

La fuente primaria El Peceño se encuentra en la planicie norte del Centro Oeste Argentino, en el actual departamento de San Rafael (provincia de Mendoza, Argentina). Se ubica próxima al volcán Nevado (3810 msnm) que se destaca en el paisaje de planicie por su elevación. El cerro Peceño posee una altura de 1707 msnm y cubre un área de 4,5 km² (Figura 1A-C). Presenta cárcavas y cursos temporarios de agua que transportan material hacia bañados estacionales en un área aproximada de $40 \mathrm{~km}^{2}$. Peceño, junto con los cerros De Afuera y Los Cerritos corresponden a asomos de la Formación Cortaderas del Mioceno superior (Figura 1B, Sepúlveda et al. 2007). Está integrada por rocas extrusivas tales como volcanitas andesíticas, dacíticas y riolíticas. Su ambiente de formación corresponde a eventos volcánicos de retroarco del Mio-Plioceno, que fueron paralelos al volcanismo de arco de la Cordillera Principal (Sepúlveda et al. 2007).

En el año 2004 la información suministrada por pobladores locales, facilitó el acceso al sector norte de cerro Peceño donde se pudo confirmar la ubicación primaria del afloramiento (Neme, comunicación pers.). Previo a esto sólo se había documentado la disponibilidad secundaria de obsidiana en cárcavas y arroyos transitorios cercanos a la fuente (Durán et al. 2004). Tras los estudios geoquímicos realizados sobre artefactos arqueológicos se conoció la existencia de dos sub-fuentes, que fueron denominadas El Peceño-1 (EP-1) y El Peceño-2 (EP-2). En el año 2009 relevamientos asistemáticos en el sector suroeste del cerro permitieron una mejor caracterización geoquímica de ambas señales, sin avanzar sobre la posición espacial. Desde el punto de vista geoquímico, la subdivisión de los grupos químicos está basada en la concentración diferencial de circonio (Zr) (Glascock 2010). En 2015 se comenzó con el muestreo espacial sistemático del cerro Peceño con el objetivo de avanzar con la caracterización espacial, arqueológica y geoquímica de la fuente de aprovisionamiento.

\section{Metodología de muestreo}

Los estudios de campo llevados a cabo en la fuente tuvieron como objetivos: a-delimitar la extensión espacial de la fuente primaria El Peceño; b-demarcar el espacio de transporte secundario de la materia prima y; c-localizar posibles áreas de producción lítica y/o actividades 


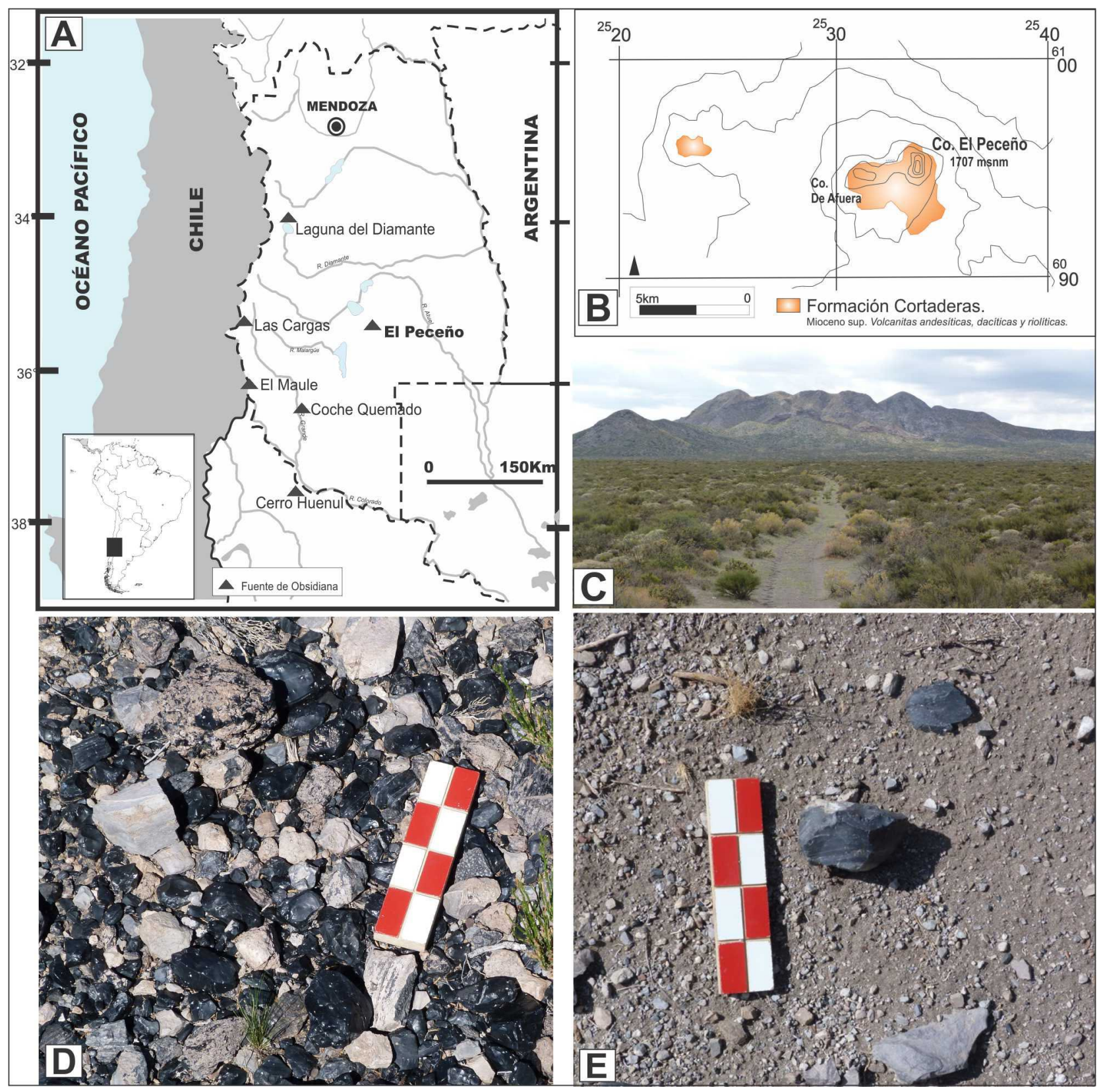

Figura 1. Localización de El Peceño. Referencias: A. Ubicación de fuentes de obsidiana en Mendoza; B. Planta de cerro Peceño y características geológicas según Sepúlveda et al. 2007; C. Vista de Cerro Peceño; D. Obsidiana de la sub-fuente El Peceño-1; E. Obsidiana de la sub-fuente El Peceño-2.

Figure 1. Location of El Peceño. References: A. Location of obsidian sources from Mendoza; B. Plant of cerro Peceño and geological characteristics according to Sepúlveda et al. 2007; C. View of Cerro Peceño; D. El Peceño-1 obsidian sub-source; E. El Peceño-2 obsidian sub-source.

múltiples. Para ello, se propuso utilizar dos herramientas metodológicas de muestreo espacial: transectas lineales y cuadrículas de recolección superficial. Las primeras tienen como fin registrar las actividades tecnológicas frecuentes en la fuente, áreas de cantera taller (Callahan 1979, Elston 1992), distancia de transporte de la materia prima, variaciones macroscópicas y diferencias espaciales en cuanto a disponibilidad, calidad para la talla, tamaños y frecuencia. Se trazaron ocho líneas de transecta, cuatro en la ladera oeste del cerro en dirección este-oeste y las cuatro restantes en la ladera este, en dirección oeste-este (Figura 2). Las transectas fueron orientadas con brújula y
GPS, con una equidistancia aproximada entre sí de $20 \mathrm{~m}$. En ambas laderas del cerro, cada línea de transecta contó con un total de 10 unidades de recolección; sin embargo, la distancia entre unidades fue variable. En el oeste se marcaron unidades de recolección cada 100 m, mientras que en el este las primeras cinco unidades fueron cada $100 \mathrm{~m}$ y las siguientes cinco cada 200 m. Esta diferencia en la equidistancia de las unidades en el este se debe a la presencia de laderas abruptas continuadas por una amplia planicie con cárcavas de escurrimiento. En cada unidad se recolectó el total de material superficial de una cuadrícula de $4 \mathrm{~m}^{2}$. 


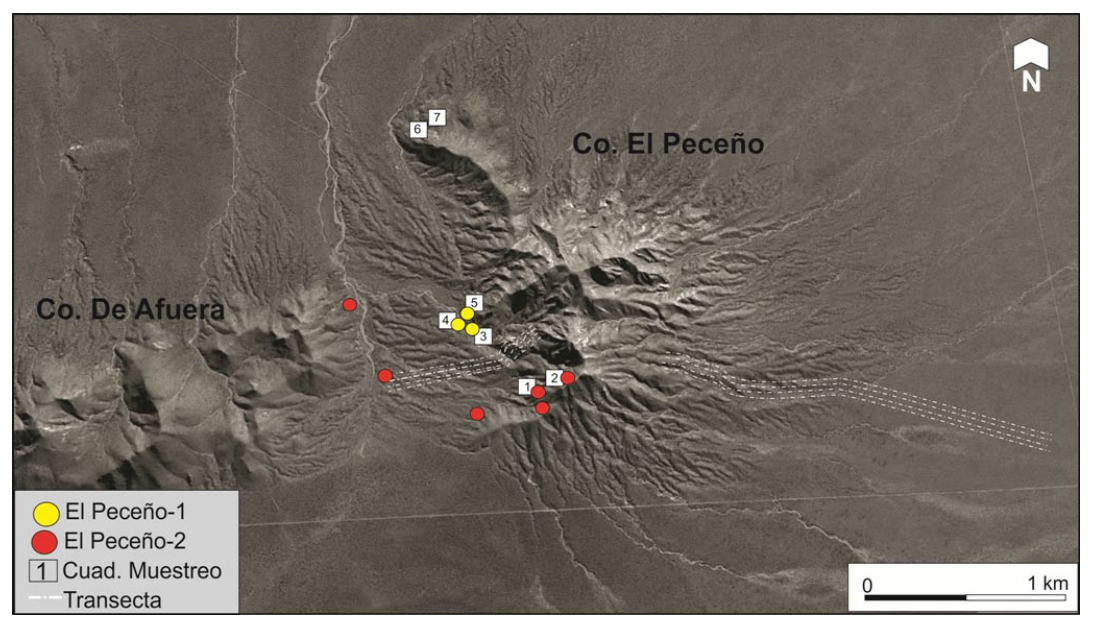

Figura 2. Muestreos superficiales y transectas realizados en El Peceño.

Figure 2. Surface sampling in El Peceño source.

La segunda herramienta propuesta corresponde a cuadrículas superficiales de muestreo. Estas tienen como objetivo relevar las actividades tecnológicas frecuentes llevadas a cabo en las áreas de taller. Luego de identificar sectores de canteo y taller sobre obsidiana, se marcaron cuadrículas de 0,50 x 0,50 m en sectores de mayor concentración de artefactos y nódulos naturales. Cada cuadrícula fue demarcada, fotografiada y ubicada su posición mediante GPS, recolectándose posteriormente el total del material superficial sin remover sedimentos. Se realizaron un total de siete unidades de muestreo, numeradas de sur a norte. Se delimitaron cinco unidades de muestreo en el oeste y dos en la ladera noroeste.

El análisis tecnológico se realizó siguiendo los lineamientos propuestos por Aschero (1975, 1983), Aschero y Hocsman (2004) y Franco $(2002,2004)$. La aptitud para la talla de la materia prima fue clasificada según la propuesta de Aragón y Franco (2007). El tamaño de cada pieza fue registrado en base a dos procedimientos. El primero fue la medición con calibre digital de los valores máximos de largo, ancho y espesor de cada artefacto (sensu Aschero 1975, 1983), entero y/o fragmentado. El segundo procedimiento fue realizado en base a la propuesta de Franco (2002) para estimar la superficie de tamaño de cada pieza. Para ello, se utiliza una grilla cuyas categorías son $5 \mathrm{~mm}$ (superficie: 5 por $5 \mathrm{~mm}$ o $5 \mathrm{~mm}^{2}$ ), $10 \mathrm{~mm}$ (superficie: 10 por $10 \mathrm{~mm}$ o $10 \mathrm{~mm}^{2}$ ), $15 \mathrm{~mm}$ (superficie: 15 por $15 \mathrm{~mm}$ o $15 \mathrm{~mm}^{2}$ ), etc., de modo que la categoría de tamaño asignada a cada artefacto corresponde a aquella cuadrícula que más se ajuste a la superficie de la pieza medida. La propuesta de Franco (2002) se considera de utilidad para procesar conjuntos con gran número de piezas, como los obtenidos en fuentes de materias primas. Por último, para el análisis de las secuencias de reducción se consideró lo propuesto por Salgán et al. (2012a y b, 2014).

\section{Análisis geoquímico}

En los muestreos se tomaron fragmentos naturales de obsidiana para su caracterización geoquímica. Al contar con antecedentes arqueométricos de la fuente El Peceño
(Giesso et al. 2011; Glascock 2010), en este trabajo sólo se aplicó la técnica no destructiva de Fluorescencia de Rayos-X (XRF). Para la selección de las muestras se tuvo en cuenta su variación macroscópica y, en los casos que fue posible, su disposición en la matriz natural o afloramiento. Se seleccionaron un total de nueve muestras de obsidiana (Tabla 1). La caracterización se llevó a cabo en el Laboratorio de Arqueometría del Research Reactor Center, Universidad de Missouri (MURR), Columbia (EE. UU.), como parte de una pasantía de intercambio de una de las autoras.

Los análisis fueron realizados con el ThermoScientific ARL Quant'X EDXRF. Este instrumento consiste en un tubo de rayos- $X$ a base de radio operado a $35 \mathrm{kV}$ y un detector de silicio termoeléctricamente enfriado. La calibración de obsidiana para XRF fue realizada usando un set de 40 muestras de fuentes de obsidiana calibradas con datos medidos previamente por ICP, XRF e INAA (Glascock y Ferguson 2012). En este estudio, cada muestra se somete a un conteo de dos minutos donde se calculan los elementos mínimos presentes. Los elementos medidos incluyen: $\mathrm{Mn}, \mathrm{Fe}, \mathrm{Zn}, \mathrm{Rb}, \mathrm{Sr}, \mathrm{Y}, \mathrm{Zr}, \mathrm{Nb}, \mathrm{Ba}$ y Th. El tratamiento de la información se realiza por medio del programa GAUSS Run-Time Module (MURR, 2012). El mismo permite generar plots bivariantes de elementos para identificar el mejor diagnóstico para XRF. En todos los casos se dibujaron elipses de un 90\% de probabilidad de confianza para indicar su rango de distribución (mayor detalle en Glascock 2008, 2011).

\section{Primeros resultados}

Los resultados del análisis geoquímico permitieron trazar la ubicación geográfica de las sub-fuentes EP-1 y EP-2 en el sector noroeste y suroeste de cerro Peceño, respectivamente. Como se observa en la Figura 2, la presencia de cárcavas en el cerro facilita el transporte de ambas variedades a distancias entre 0,80 y 1,5 km desde los afloramientos. El análisis geoquímico de los afloramientos primarios detectados hasta el momento en el cerro, permitieron confeccionar un mapa preliminar de distribución de las sub-fuentes y caracterizarlas 


\begin{tabular}{cccccccccccc}
\hline ANID & $M n$ & $F e$ & $Z n$ & $R b$ & $S r$ & $Y$ & $Z r$ & $N b$ & $B a$ & $T h$ & Fuente \\
\hline LSM235 & 900,7 & 5877,5 & 44,9 & 222,8 & 250,9 & 11,9 & 123,9 & 24,3 & 1098,8 & 10,5 & EP-1 \\
LSM236 & 933,3 & 6189,5 & 47,7 & 227,3 & 262,8 & 12,2 & 124,2 & 24,8 & 1143,4 & 11,3 & EP-1 \\
LSM237 & 918,6 & 6116,2 & 44,3 & 226,0 & 258,8 & 12,8 & 123,2 & 25,5 & 1164,2 & 13,5 & EP-1 \\
LSM238 & 799,1 & 6322,5 & 47,2 & 237,8 & 255,9 & 10,7 & 117,8 & 21,9 & 1000,2 & 12,2 & EP-1 \\
LSM239 & 1033,9 & 7176,6 & 50,7 & 234,1 & 292,4 & 13,8 & 133,7 & 25,5 & 1213,6 & 12,3 & EP-1 \\
LSM240 & 930,1 & 5637,1 & 45,8 & 258,6 & 242,5 & 9,1 & 84,9 & 23,8 & 790,6 & 9,5 & EP-2 \\
LSM241 & 854,2 & 5172,0 & 38,5 & 247,1 & 240,3 & 8,3 & 76,0 & 22,4 & 848,1 & 9,4 & EP-2 \\
LSM242 & 854,2 & 5172,0 & 38,5 & 247,1 & 240,3 & 8,3 & 76,0 & 22,4 & 848,1 & 9,4 & EP-2 \\
LSM243 & 867,5 & 5228,5 & 41,0 & 249,2 & 239,8 & 8,8 & 72,4 & 22,6 & 838,3 & 10,3 & EP-2 \\
\hline
\end{tabular}

Tabla 1. Concentración de elementos traza en partes por millón para muestras de obsidiana El Peceño, analizadas por XRF.

Table 1. Trace-elements concentration in parts per million for obsidian from El Peceño source, analyzed by XRF.

macroscópicamente (Figura 2). En EP-1 se registró un transporte de 1 a $1,5 \mathrm{~km}$ de distancia, de nódulos naturales de una superficie estimada entre 10 y $35 \mathrm{~mm}^{2}$. Los afloramientos son visibles a una distancia aproximada de $2 \mathrm{~km}$. Las variaciones macroscópicas presentan un $20 \%$ de color gris con vetas paralelas de color negro, marrón y rojo y, el 79\% restante es de color negro, brillo vítreo y presencia de impurezas o cristales. Su aptitud para la talla puede clasificarse entre buena y muy buena (Figura 1D). También se destaca un 1\% de variedades de color verde homogéneo y otra de pátina o cubierta gruesa de color marrón.

En EP-2 los nódulos registrados son de mayor tamaño, variando entre 15 a $40 \mathrm{~mm}^{2}$. Su dispersión espacial oscila entre 0,80 y $1 \mathrm{~km}$ de la fuente, los afloramientos se encuentran cubiertos por sedimentos y no se observan a la distancia. En esta sub-fuente las variaciones macroscópicas comprenden a una obsidiana negra de brillo vítreo $(40 \%)$ y otra de color gris $(17 \%)$, con variedades gris claro (14\%) y gris opaco (17\%). Su calidad para la talla puede clasificarse como muy buena (Figura 1E). En este sector del cerro Peceño también se encuentra disponible en forma primaria y secundaria, una roca dacítica de buenas aptitudes para la talla, observándose también signos de aprovisionamiento y reducción de esta materia prima.

Desde la perspectiva tecnológica, se analizaron hasta el momento un total de 821 artefactos de obsidiana (99\%) y dacita (1\%), correspondientes a tres cuadrículas de muestreo superficial en el sector noroeste (M3, M4 y M5) atribuidas a la variedad EP-1 y tres líneas de transecta en el suroeste (TRO1, TRO2 y TRO3) que se corresponden con la variedad EP-2, ambos ubicados en la ladera oeste de cerro Peceño. Las muestras fueron atribuidas a EP-1 y EP-2 en base a su ubicación espacial y a sus características macroscópicas antes descriptas. Si bien se aplicaron distintas herramientas de muestreo en cada sub-fuente, los resultados permiten generar una tendencia de actividades llevadas a cabo en cada sector de la ladera oeste. En las cuadrículas de muestreo de EP-1 se registraron 699 piezas, de las cuales el $49 \%$ corresponde a artefactos y el $51 \%$ a nódulos y fragmentos naturales. En clases artefactuales, el 57\% corresponde a artefactos no diferenciados, en su mayoría por fractura o atributos dudosos. Le siguen en frecuencia el $36 \%$ de desechos de talla, $6 \%$ de núcleos y el $0,1 \%$ de artefactos formatizados. En desechos de talla (n: 133), el $83 \%$ se encuentra fracturado y el $17 \%$ entero. En desechos enteros las lascas internas (lascas de arista y angulares) representan el 98\% de la muestra, seguida por un $2 \%$ de lascas externas (primarias, secundarias y de reactivación de núcleo). El $50 \%$ de los desechos presenta un remanente de corteza que cubre hasta un $25 \%$ de la cara dorsal, mientras que el $50 \%$ restante no presenta corteza. En núcleos (n: 20), predominan las morfologías amorfas o no diferenciadas y sólo un núcleo pudo ser clasificado como piramidal. El $40 \%$ presenta entre el 25 y $50 \%$ de su superficie cubierta con corteza y el $60 \%$ restante no tiene corteza. En estos últimos se destaca la presencia de una pátina superficial continua. Las superficies de tamaño de los artefactos oscilan entre 20 y $40 \mathrm{~mm}^{2}(55 \%)$, seguido por superficies comprendidas entre 50 y $75 \mathrm{~mm}^{2}$ (40\%) y de $15 \mathrm{~mm}^{2}$ (5\%). El único registro de artefactos formatizados corresponde a un artefacto unifacial que fue atribuido a una preforma de cabezal lítico (largo: $26 \mathrm{~mm}$, ancho: 29,1 $\mathrm{mm}$ y espesor: $6 \mathrm{~mm}$ ).

En las transectas realizadas en el área cercana a los afloramientos de EP-2 se registraron 122 piezas; de ellos el $61 \%$ corresponde a artefactos y el $39 \%$ a nódulos y fragmentos naturales. Entre los artefactos, las clases predominantes son los desechos de talla (83\%), seguidas por núcleos (16\%) y artefactos formatizados (1\%). Entre los desechos de talla el $85 \%$ se presenta entero y el $15 \%$ restante fracturado. En desechos enteros el 62\% corresponde a lascas externas y el $38 \%$ a lascas internas. 
Entre las lascas externas el 58\% presenta remanente de corteza que cubre entre el 75 y el $100 \%$ de la cara dorsal y el $42 \%$ restante el $25 \%$ de su cara dorsal. Respecto a los núcleos (n: 12), predominan las morfologías amorfas (42\%), seguido por núcleos bipolares (33\%), los sub piramidales (17\%) y los globulosos (8\%). El $42 \%$ presenta corteza que cubre entre el 25 y el $50 \%$ de su superficie externa y un $58 \%$ no posee corteza. La presencia de pátina es variable y se encuentra en nódulos naturales y en los negativos de lascado del $51 \%$ de los núcleos. Los tamaños frecuentes poseen una superficie estimada entre 20 y $35 \mathrm{~mm}^{2}$ (67\%), seguido por tamaños entre 40 y $50 \mathrm{~mm}^{2}(25 \%)$ y $15 \mathrm{~mm}^{2}$ (8\%). El artefacto formatizado documentado en la transecta TRO3, corresponde a una herramienta bifacial confeccionada en dacita gris. La misma posee una longitud de 152,1 mm, un ancho de $82,5 \mathrm{~mm}$ y un espesor de $18,2 \mathrm{~mm}$, y fue clasificada como una raedera; aún se desconoce qué función pudo cumplir en el contexto de la fuente.

\section{Discusión}

El muestreo espacial y geoquímico de El Peceño permite proponer que EP-1 presenta una distribución espacial mayor en relación con EP-2. Los nódulos naturales registrados en el sector de EP-2 presentan tamaños mayores a EP-1; sin embargo, los afloramientos de esta última variedad son visibles desde puntos distantes. Con respecto a las etapas de la secuencia de reducción representadas, en EP-1 se registraron actividades de extracción de lascas y confección de preformas. Los núcleos no presentan morfología definida -a excepción de uno piramidal- y las superficies de tamaño estimado sugieren su descarte no agotado. La baja reserva de corteza que presenta la materia prima en EP-1 pudo favorecer el aprovechamiento y traslado de nódulos enteros, sin modificación. Esta propuesta podrá ser cotejada con la información tecnológica regional. En EP-2 y a diferencia de EP-1, los desechos de talla frecuentes son las lascas externas lo que indicaría que aquí habrían sido más frecuentes las tareas de descortezamiento. La presencia de núcleos con morfología definida, puede responder a la búsqueda y selección de soportes, lascas y/o formas base particulares. Este último planteo se refuerza al considerar que EP-2 presenta aptitud para la talla muy buena, sin inclusiones internas y escasa presencia de pátina en su superficie externa.

Dadas las diferencias observadas en los registros analizados y pese a los escasos metros de distancia que separan a las dos variedades, se puede considerar que existen diferencias en las formas de obtención de obsidiana en las sub-fuentes. Esto permite generar tendencias y expectativas en cuanto a sus modos de transporte y uso. En el caso de EP-1, se espera que sea frecuente su traslado como lascas o preformas de superficies no mayores a los $75 \mathrm{~mm}$. El traslado también podría incluir a los nódulos naturales que presentan pátinas como corteza. Respecto a EP-2, se espera que prime el traslado de núcleos o preformas, haya registro de desechos con mayor reserva de corteza en relación a EP-1, y aptitud para la talla muy buena, con baja presencia de impurezas.

En la Tabla 2 se observa la frecuencia de artefactos de obsidiana con señal geoquímica asignada a la fuente El Peceño (n: 246), procedentes de sitios ubicados a distancias comprendidas entre 0 y $260 \mathrm{~km}$ de la fuente, y discriminados según pertenezca a la sub-fuente EP-1 o EP2. Las muestras incluidas en la tabla han sido analizadas en el MURR por el método XRF y su información química se encuentra disponible en Giesso et al. (2011), Cortegoso et al. (2012), Salgán et al. (2012a, 2014) y en la Tabla 1 de este trabajo. La información fue agrupada según los rangos de distancia propuestos por Civalero y Franco (2003) y Cortegoso et al. (2012). Se indica en cada rango, el número de sitios donde fueron registrados. Aquellos sitios con distancias superiores a los $100 \mathrm{~km}$ lineales a la fuente, se agruparon según rangos de $50 \mathrm{~km}$. La muestra incluida en la tabla, está compuesta en la mayoría de los casos, por piezas enteras y formatizadas (Cortegoso et al. 2012; Giesso et al. 2011), procedentes de conjuntos

\begin{tabular}{|c|c|c|c|c|c|c|}
\hline \multicolumn{2}{|c|}{ Rangos de distancia (km) } & \multirow{2}{*}{$\begin{array}{c}\text { EP-1 } \\
-\end{array}$} & \multirow{2}{*}{$\begin{array}{c}\text { No Sitios } \\
-\end{array}$} & \multirow{2}{*}{$\begin{array}{c}\text { EP-2 } \\
-\end{array}$} & \multirow{2}{*}{$\begin{array}{c}\text { No }^{\circ} \text { Sitios } \\
-\end{array}$} & \multirow{2}{*}{$\begin{array}{c}\text { Total Sitios } \\
-\end{array}$} \\
\hline & $0-5$ & & & & & \\
\hline Obsidiana & $5-10$ & $35 \%(69)$ & 8 & $2 \%(1)$ & 1 & 9 \\
\hline & $30-40$ & $38 \%(76)$ & 2 & $17 \%(8)$ & 1 & 3 \\
\hline \multirow{5}{*}{$\begin{array}{l}\text { Obsidiana } \\
\text { no local }\end{array}$} & $40-70$ & $3 \%(6)$ & 3 & $29 \%(14)$ & 5 & 5 \\
\hline & $70-100$ & $13 \%(25)$ & 8 & $40 \%(19)$ & 5 & 12 \\
\hline & $100-150$ & $2 \%(4)$ & 2 & $13 \%(6)$ & 4 & 5 \\
\hline & $150-200$ & - & - & - & - & - \\
\hline & $>200$ & $9 \%(18)$ & 3 & - & - & 3 \\
\hline \multicolumn{2}{|c|}{ Total } & 198 & & 48 & & 37 \\
\hline
\end{tabular}

Tabla 2. Frecuencias de muestras de obsidianas de las sub-fuentes El Peceño-1 (EP-1) y El Peceño-2 (EP-2) según rangos de distancia y número de sitios donde fueron registradas. Referencias: "()" entre paréntesis se indica el número total de muestras con señal geoquímica EP-1 y EP-2 para cada rango.

Table 2. Frequencies of obsidian samples of sub-sources El Peceño-1 (EP-1) and El Peceño-2 (EP-2) for distance ranges and number of sites where they were registered. Reference: "()" in brackets the total number of signal samples geochemistry EP-1 and EP-2 is indicated for each range. 
estratigráficos (21) y superficiales (16). La totalidad de los conjuntos estratigráficos fueron asignados por medio de datación radiocarbónica al Holoceno tardío (Cortegoso et al. 2012; Salgán et al. 2012b), mientras que los conjuntos superficiales no poseen información temporal y han sido atribuidos tentativamente a momentos tardíos (Neme y Gil 2012).

En la tabla se destaca un mayor traslado y uso de EP-1 (80\%) respecto a EP-2 (20\%). Del total de sitios donde se identifican (n: 37), el 70\% presenta EP-1 y el $43 \%$ EP-2. Con respecto a los rangos de distancia espacial, se destaca que EP-1 presenta mayor número de muestras en los rangos de distancia locales -mayor a $5 \mathrm{~km}$ y menor a $40 \mathrm{~km}-$, mientras que EP-2 incrementa su frecuencia en distancias consideradas no locales, ubicadas a más de $40 \mathrm{~km}$. Sin embargo, EP-1 también presenta registros en sitios ubicados a distancias mayores a los $200 \mathrm{~km}$, los que incluyen artefactos del sureste de San Luis (Heider 2015) y norte de Mendoza (Salgán 2015). En el análisis de los sitios, es de relevancia destacar que ambas variedades coinciden sólo en dos de los 10 sitios ubicados en los rangos de distancia local y en cuatro de los 27 sitios ubicados en el rango de las no locales.

\section{Conclusión y perspectivas}

El Peceño es la única fuente de obsidiana ubicada en la planicie norte del Centro Oeste Argentino y se considera la tercera en importancia de la región (Cortegoso et al. 2012). Los antecedentes geoquímicos, sumados a los estudios preliminares presentados aquí, permiten proponer un uso predominante de EP-1 en los rangos de distancia local, diferente de la variedad EP-2, la cual es más frecuente a distancias no locales. Esto podría responder a las características que presenta la materia prima en la fuente y puede ser explicado por los resultados aquí expuestos. En EP-2 son frecuentes las actividades de descortezamiento y reducción de núcleos. Estos últimos tienen superficies de tamaño mayor en relación a EP-1 y aptitudes muy buenas para la talla. Por este motivo, EP-2 pudo haber sido seleccionada para su traslado, mientras que la variedad EP-1 -de mayor oferta en el espacio- fue destinada al uso local. Para poder discutir en profundidad las tendencias expuestas, es necesario avanzar en el estudio tecnológico de los sitios en los cuales se registra su uso, así como establecer parámetros de análisis tecnológico y de muestreo que permitan comparar los resultados.

Si bien los estudios regionales disponibles ubican el registro temporal de El Peceño en el Holoceno tardío, sería de interés poder caracterizar la cronología de los conjuntos superficiales con métodos alternativos como la hidratación de obsidiana. Los próximos pasos de investigación buscan avanzar con la caracterización tecnológica y geoquímica de El Peceño, así como de los conjuntos arqueológicos locales y no locales. Este aporte forma parte de un proyecto mayor que propone el abordaje sistemático de las fuentes del sur de Mendoza y su articulación con los proyectos regionales en marcha.

San Rafael, 20 de febrero de 2016

\section{Agradecimientos}

Agradecemos especialmente a Michel D. Glascock y al Laboratorio de Arqueometría del MURR, por la colaboración y entrenamiento en el análisis geoquímico de las piezas arqueológicas. A Gustavo Neme, Federico Bobillo y Guillermo Heider por su colaboración y comentarios durante los trabajos de campo. A Pedro Serra y familia, por facilitarnos la estadía durante las tareas de campo. A Adolfo Gil y Gustavo Neme por estimular nuestras investigaciones en el área. A CONICET y ANPCYT, por financiar nuestros trabajos de investigación. A los evaluadores anónimos que con sus sugerencias y comentarios colaboraron en mejorar significativamente este manuscrito. Este trabajo fue realizado en el marco de los proyectos PICT 2012-1015 y PICT 2014-3270. Los errores $u$ omisiones del presente trabajo son responsabilidad de las autoras.

\section{Bibliografía}

Aragón, E. \& N. Franco. 1997. Características de rocas para la talla por percusión y propiedades petrográficas. Anales del Instituto de la Patagonia 25: 187-199.

Aschero, C. 1975. Ensayo para una clasificación morfológica de artefactos líticos aplicados a estudios tipológicos comparativos. Informe a CONICET. Ms.

Aschero, C. 1983. Ensayo para una clasificación morfológica de artefactos líticos aplicada a estudios tecnológicos comparativos. Apéndice A-C. Revisión 1983. Cátedra de Ergología y Tecnología. Facultad de Filosofía y Letras. Universidad de Buenos Aires. Ms.

Aschero, C. \& S. Hocsman. 2004. Revisando cuestiones tipológicas en torno a la clasificación de artefactos bifaciales. A. Acosta, D. Loponte y M. Ramos (Eds.), Temas de Arqueología, Análisis Lítico, pp. 7-25, Universidad Nacional de Luján, Buenos Aires.

Barberena. R., A. Hajduk, A. Gil, G. Neme, V. Durán, M. Glascock, M. Giesso, K. Borrazzo, M. P. Pompei, M. L. Salgán, V. Cortegoso, G. Villarosa \& A. A. Rughini. 2011. Obsidians in the southcentral Andes: geological, geochemical, and archaeological assessment of north Patagonian sources (Argentina). Quaternary International 245(1): 25-36.

Callahan, E. 1979. The basics of biface knapping in the eastern fluted point tradition. A manualfor flintknappers and lithic analysts. Archaeology of Eastern North America 7: 1-180.

Civalero, M. T. \& N. V. Franco. 2003. Early human occupations in Western Santa Cruz province, southernmost South America. Quaternary International 109-110: 77-86.

Cortegoso, V., G. Neme, M. Giesso, V. Durán \& A. Gil. 2012. El uso de la obsidiana en el sur de Mendoza. G. Neme \& A. Gil 
(Comps.), Paleoecología humana en el sur de Mendoza, pp. 180-211, Sociedad Argentina de Antropología, Buenos Aires.

Cortegoso, V., R. Barberena, V. Durán \& G. Lucero. 2016. Geographic vector of human mobility in the Andes (34-36 S): Comparative analysis of 'minor' obsidian sources. Quaternary International, 422 (1): 81-92

Durán, V., M. Giesso, M. Glascock, G. Neme, A. Gil \& L. Sanhueza. 2004. Estudio de fuentes de aprovisionamiento y redes de distribución de obsidiana durante el Holoceno tardío en el sur de Mendoza (Argentina). Estudios Atacameños 28: 25-43.

Eerkens, J., Vaughn, K., Linares-Grados, M., Conlee C., Schreiber, K., Glascock, M. D. \& N. Tripcevich. 2009. Spatio-temporal patterns in obsidian consumption in the Southern Nasca Region, Peru. Journal of Archaeological Science 37: 825-832.

Elston R. G. 1992. Modeling the economics and organization of lithic procurement. En: R. G. Elston y R. Raven (Eds.), Archaeological Investigations at Tosawhi, a great basin quarry, II Vol. Roport, Intermountain Research.

Ericson, J. 1977. Egalitarian exchange systems in California: a preliminary view. Timothy Earle y Jonathan Ericson (Eds.), Exchange Systems in Prehistory, pp. 109-26, Academic Press, Nueva York.

Franco, V. N. 2002. Estrategias de utilización de recursos líticos en la cuenca superior del río Santa Cruz. Tesis doctoral inédita. Universidad de Buenos Aires.

Franco, V. N. 2004. La organización tecnológica y el uso de escalas espaciales amplias. El caso del sur y oeste de Lago Argentino. A. Acosta, D. Loponte y M. Ramos (Eds.), Temas de arqueología. Análisis lítico, pp. 101-144, Luján, Universidad Nacional de Luján.

Giesso, M., Durán, V., Neme, G.A., Glascock, M.D., Cortegoso, V., Gil, A.F. \& L. Sanhueza. 2011. A study of obsidian source usage in the central Andes of Argentina and Chile. Archaeometry 53 (1): 1-21.

Glascock, M. D. 2008. Archaeometry. D. M. Pearsall (Ed.), Encyclopedia of Archaeology, pp. 489-494, Oxford: Academic.

Glascock, M. D. 2010. X-ray Fluorescence Analysis of Obsidian from Argentina. Archaeometry Laboratory Report. Ms.

Glascock, M. D. 2011. Comparison and Contrast between XRF and NAA: Used for Characterization of Obsidian Sources in Central Mexico. M. S. Shackley (Ed.), X-Ray Fluorescence Spectrometry (XRF) in Geoarchaeology, pp. 161-192, Springer, New York.

Glascock, M. D. \& J. R. Ferguson. 2012. Report on the Analysis of Obsidian Source Samples by Multiple Analytical Methods. Archaeometry Laboratory at MURR, University of MissouriColumbia.

Gould, R.A. \& S. Saggers. 1985. Lithic Procurement in Central Australia: A Closer Look at Binford's Idea of Embeddedness in Archaeology. American Antiquity 50 (1): 117-136.
Heider, G. 2015. “Los pueblos originarios en el Norte de Pampa Seca. Una mirada arqueológica a los cazadores recolectores del Sur de las provincias de Córdoba y San Luis, Argentina". Facultad de Filosofía y Humanidades, Universidad Nacional de Córdoba, Córdoba, Argentina, 423 pp. Tesis doctoral inédita.

Lagiglia, H. 2002. Arqueología prehistórica del sur mendocino y sus relaciones con el Centro Oeste Argentino. A. Gil y G. Neme (Eds.), Entre Montañas y Desiertos: Arqueología del Sur de Mendoza, pp.43-64, Sociedad Argentina de Antropología, Buenos Aires.

Neme, G. \& A. Gil. 2008. Biogeografía humana en los andes meridionales: tendencias arqueológicas en el sur de Mendoza. Chungara, Revista de Antropología Chilena 40 (1): 5-18.

Neme, G. \& A. Gil. 2012. El registro arqueológico del sur de Mendoza en perspectiva biogeográfica. G. Neme \& A. Gil (Comps.), Paleoecología humana en el sur de Mendoza: perspectivas arqueológicas, pp. 255-280, Sociedad Argentina de Antropología, Buenos Aires.

Salgán, M. L., A. Gil \& G. Neme. 2012a. Obsidianas en La Payunia (sur de Mendoza, Argentina): patrones de distribución e implicancias en la ocupación regional. Magallania, 40(1): 263-277.

Salgán, M. L., S. Paulides \& V. Cortegoso. 2012b. Rocas, rangos de acción y biogeografía humana en el sur de Mendoza. G. Neme y A. Gil (Comps.), Paleoecología humana en el sur de Mendoza: perspectivas arqueológicas, pp. 157-180, Sociedad Argentina de Antropología, Buenos Aires.

Salgán, M. L., A. Gil \& G. Neme. 2014. Movilidad, aprovisionamiento y uso de obsidiana en El Payén, sur de la provincia de Mendoza, Argentina. Comechingonia 18(1): 33-50.

Salgán, M. L. 2015. X-Ray Fuorescence Analysis Of Obsidian From Mendoza, Argentina. Archaeometry Laboratory Final Report. Ms.

Salgán, M. L., R. Garvey, G. Neme, A. Gil, M. Giesso, M. Glascock \& V. Durán. 2015. Las Cargas: Characterization of a Southern Andean Obsidian Source and Its Prehistoric Use. Geoarchaeologay. An International Journal 30(2): 139-150.

Seelenfreud, A. C., Roes, R., Bird, G., Bailey, R., Bárcena \& V. Durán. 1996. Trace-Element Analysis of Obsidian Sources and Artifacts of Central Chile (Maule River Basin) and Western Argentina (Colorado River). Latin American Antiquity 7(1): 7-20.

Sepúlveda, E., A. Bermúdez, O. Bordonaro \& D. Delpino. 2007. Hoja Geológica 3569-IV, Embalse El Nihuil, provincia de Mendoza. Instituto de Geología y recursos minerales, Servicio Geológico Minero Argentino, Boletín 268: 52.

Summerhayes, G.R., Kennedy, J., Matisoo-Smith, E., Mandui, H., Ambrose, W., Allen, C., Reepmeyer, C., Torrence, R. \& F. Wadra. 2014. Lepong: A new obsidian source in the Admiralty Islands, Papua New Guinea. Geoarchaeology. An International Journal 29: 238-248. 